\title{
Achtsamkeit in Organisationen
}

Telse A. Iwers ${ }^{1}$. Eva-Maria Schulte ${ }^{2}$

Online publiziert: 22. Mai 2018

๑) Springer Fachmedien Wiesbaden GmbH, ein Teil von Springer Nature 2018
Das Konzept der Achtsamkeit wird seit einigen Jahren aus verschiedenen Perspektiven intensiv diskutiert und beforscht. Es verliert dabei kaum an Beachtung, wenngleich sich die Schwerpunkte der Befassung leicht verschieben. So hat z.B. die Gruppendynamik und Organisationsberatung (der Vorläufer unserer Zeitschrift) im Jahr 2014 ein Themenheft zur Achtsamkeit herausgebracht. Hier lagen die Schwerpunkte der Betrachtung in individuellen Wirkweisen von Beratungsansätzen, Interventionen und Bildungstrainings. Das nunmehr vorliegende Heft verschiebt den Schwerpunkt in Richtung interaktionaler, kollektiver und organisationaler Wirkmechanismen. Kollektive Achtsamkeit wird dabei als sozialer Prozess verstanden und kann eine mögliche Antwort auf organisationale Herausforderungen, wie Termin- und Leistungsdruck, Unterbrechungen der Arbeit und hohe Veränderungsdynamiken, sein. Diese gehen oft einher mit einer zunehmenden Beanspruchung von Mitarbeitenden. Achtsamkeit in Organisationen kann diesem negativen Trend entgegenwirken. Denn vergleichbar zur individuellen Ebene ist die erhöhte Aufmerksamkeit auf das Hier und Jetzt auch ein zentraler Bestandteil kollektiver Achtsamkeit: Statt vorschneller, routinierter Reaktionen zeichnen sich achtsame Organisationen dadurch aus, dass sie auf allen organisationalen Ebenen aktuelle Situationen und Bedingungen bewusst wahrnehmen und auf diese als Kollektiv flexibel und angemessen reagieren (Sutcliffe et al. 2016). Dieses Themenheft beleuchtet daher sowohl die Frage der Relevanz der individuellen Achtsamkeit im organisationalem Setting als auch die Frage nach der Konzeptualisierung, Wirkung und Förderung kollek-

Prof. Dr. Telse A. Iwers, MHEd.

GIO@Iwers-Stelljes.de

Dr. Eva-Maria Schulte

e.schulte@tu-braunschweig.de

1 Fakultät für Erziehungswissenschaft, Universität Hamburg, Hamburg, Deutschland

2 Lehrstuhl für Arbeits-, Organisations- und Sozialpsychologie, Technische Universität Braunschweig, Braunschweig, Deutschland tiver Achtsamkeit auf organisationaler Ebene sowie auf Teamebene.

Der theoriegeleitete Beitrag von Gebauer und Brückner stellt zunächst die beiden Konzepte der individuellen und kollektiven Achtsamkeit gegenüber. Darauf aufbauend wird die gleichzeitige Berücksichtigung der individuellpsychischen Ebene (bspw. Techniken der individuellen Achtsamkeit zur An-/Entspannung oder Fokussierung/ Ungerichtetheit), der Interaktionsebene (bspw. Einüben neuer Muster im Team) und der Organisationsebene (bspw. Veränderung von Entscheidungsprämissen) für die Förderung der organisationalen Achtsamkeit, herausgearbeitet. Die Autor/-innen betonen, dass die bisherige einseitige Fokussierung auf individuelle Achtsamkeitstrainings daher zukünftig durch den expliziten Einbezug organisationaler Ebenen erweitert werden muss.

Schulte, Steffens, Kauffeld und Höller argumentieren, dass ein Grund für die bisherige starke Fokussierung auf die individuelle Ebene in Forschung und Praxis auch darin liegt, dass es an geeigneten Messinstrumenten der Achtsamkeit auf der Ebene des Teams fehlt. Basierend auf Arbeiten zur kollektiven Achtsamkeit auf organisationaler Ebene sowie Interviews, entwickeln die Autor/-innen daher ein Instrument zu Messung der Achtsamkeit im Team und diskutieren die Anwendung in der Praxis anhand eines Beispiels.

Soucek, Ziegler, Schlett und Pauls untersuchen in ihrem Beitrag die Relevanz der Achtsamkeit auf individueller und organisationaler Ebene und beleuchten dabei sowohl den direkten Einfluss auf resilientes Verhalten bei der Arbeit als auch den indirekten Einfluss auf die psychische Gesundheit und das Arbeitsengagement. Ihre Befunde belegen dabei den inkrementellen Einfluss der organisationalen zur individuellen Achtsamkeit, so dass die Autor/-innen einen kombinierten Ansatz von Verhaltens- (individuelle Achtsamkeit) und Verhältnisprävention (organisationale Achtsamkeit) nahelegen.

Lange, Bormann und Rowold fokussieren auf die Rolle der Führungskraft im Kontext organisationaler Achtsamkeit: Während bisherige Forschungen die negativen Effekte von als destruktiv wahrgenommener Führung sowie die po- 
sitiven Effekte transformationaler Führung belegen, gibt es wenig Untersuchungen zu den Antezedenzien dieser Führungsstyle. Die Autor/-innen argumentieren, dass die individuelle Achtsamkeit der Führungskraft einen direkten Einfluss auf ihr Führungsverhalten hat und bestätigen empirisch, dass achtsame Führungskräfte von ihren Mitarbeitenden als weniger destruktiv und mehr transformational wahrgenommen werden.

Krohn zeigt in seinem Beitrag „Ärztliche Resilienz durch Achtsamkeit“ zunächst die Veränderungen im deutschen Gesundheitssystem hin zu einem marktorientierten managementgesteuerten System auf und leitet anschließend vor dem Hintergrund der in diesem Bereich ohnehin schon gegebenen Belastungsfaktoren deren zunehmende Verstärkung auf. Dieser Entwicklung kann durch den Einsatz von resilienzfördernden Achtsamkeitsmeditationen begegnet werden, die zugleich einen Beitrag zur Entwicklung von „Spiritual Care“ leisten können. Damit wirken sie nicht nur auf das im medizinischen Bereich tätige Subjekt, sondern auch auf die Interaktion im Team wie auch mit Patient/ -innen.

Altner, Erlinghagen, Körber, Cramer und Dobos fokussieren in Ihrem Beitrag die Frage, wie basierend auf einer Haltungs- und Verhaltensänderung einzelner Personen eine achtsame Organisationsentwicklung entstehen kann. Dafür berichten die Autor/-innen erste qualitative Ergebnisse einer Intervention zur Steigerung der Achtsamkeit in Grundschulen. Unter Einbezug der vier Ebenen (1) Lehrende selbst, (2) Interaktionen zwischen Lehrenden, (3) Interaktionen mit Schüler/-innen sowie (4) der organisationalen Ebene leiten die Autor/-innen relevante Faktoren zur Stärkung organisationaler Achtsamkeit ab.

Insgesamt befassen sich die vorliegenden Beiträge mit verschiedenen Perspektiven auf Achtsamkeit im organisationalen Kontext. Es werden theoretische Konzeptionen ebenso präsentiert wie konkrete Anwendungen und deren Evaluation. Dabei werden die Positionierungen zwischen individueller und organisationaler Orientierung graduell unterschiedlich aufgespannt. Damit verdeutlichen die Ar- beiten in ihrer Gesamtschau die vielfältigen Wirkzusammenhänge von Achtsamkeit zwischen Person und Organisation.

\section{Literatur}

Sutcliffe, K. M., Vogus, T. J., \& Dane, E. (2016). Mindfulness in Organizations: A Cross-Level Review. Annual Review of Organizational Psychology and Organizational Behavior, 3, 55-81. https:// doi.org/10.1146/annurev-orgpsych-041015-062531

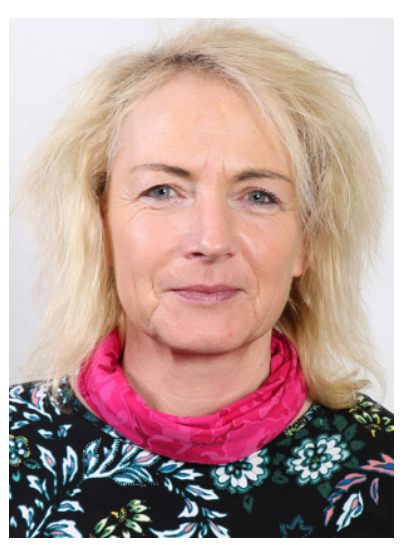

Prof. Dr. Telse A. Iwers Erziehungswissenschaftlerin, Gestalttherapeutin und Supervisorin. Universität Hamburg, Fakultät für Erziehungswissenschaft. Forschungsschwerpunkte: Führungs- und Aufstiegskompetenz von Frauen; Professionalisierung durch Beratung und Coaching im Lehramt; mentale Blockaden und Prozesse deren Lösung durch Selbstregulation; Entwicklung von Achtsamkeit durch Introvision.

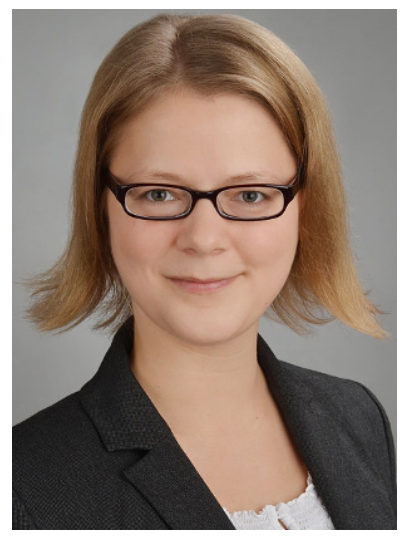

Dr. Eva-Maria Schulte ist seit 2009 wissenschaftliche Mitarbeiterin am Lehrstuhl für Arbeits-, Organisations- und Sozialpsychologie an der Technischen Universität Braunschweig. Ihre Forschungsschwerpunkte umfassen Teamarbeit, Coaching, Gesundheit, Führung sowie die Evaluation von Interventionen. Seit 2016 ist sie zudem als Senior Consultant bei der 4A-SIDE GmbH tätig (Schwerpunkte Training und Coaching). 